\title{
Review: falls can be prevented in older people, but interventions should be multifaceted and targeted
}

Feder G, Cryer C, Donovan S, et al. on behalf of the Guidelines'Development Group. Guidelines for the prevention of falls in people over 65. BMJ 2000 Oct 21;321:1007-11.

\section{QUESTION: What is the evidence that risk factor modification and other interventions will reduce falls in people $\geqslant 65$ years of age?}

\section{Data sources}

Studies were identified by searching Medline to March 1998 with the terms fall, falls, accidental falls, fracture, elderly, aged, older, and senior. Bibliographies of studies and reviews were scanned, and experts were contacted.

\section{Study selection}

Randomised controlled trials (RCTs) were selected if the interventions were designed to minimise or prevent exposure to risk factors for falling or fractures; participants were $\geqslant 65$ years of age and living in the community or in residential care; and the number of falls, fractures, or people who had fallen was reported.

\section{Data extraction}

Data were extracted on study quality, patient characteristics, settings, types of interventions (exercise and multifaceted), and outcomes. Recommendations were graded (A for evidence from multiple RCTs or metaanalyses; B for a single RCT or weak, inconsistent findings from multiple RCTs; and $\mathrm{C}$ for limited evidence).

\section{Main results}

All trials were under powered to detect differences in injurious falls. 8 trials evaluated an exercise intervention alone. 3 of these studies included high risk participants, and 2 showed a decreased risk for falling. In the 5 studies of unselected participants, only 1 trial of Thai chi (balance training) showed a reduction in the number of falls (grade B evidence). Pooling of 4 of these trials did not show a reduction in falls. Another trial of brisk walking in women with osteoporosis showed that falls were increased in the walking group.

5 published trials and 1 unpublished trial evaluated multifaceted interventions, and all showed decreased risks for falling (grade B evidence). Programmes that combine interventions reduce falls (grade A evidence). Those that emphasise assessment of postural hypotension, number of drugs, balance, transfer, and gait are successful (grade B evidence).

Reanalysis of some of the trials showed that home based interventions were not as effective as programmes in residential settings and did not reduce falls (grade A evidence). Patients who come to the emergency departments after falls have a lower frequency of subsequent falls after medical and occupational follow up and referral (grade B evidence). People in residential settings also benefit from targeted interventions. For example, padded hip protectors reduce fractures in high risk patients. No economic evaluations were available.

\section{Conclusions}

Falls can be prevented in older people. The most effective interventions are multifaceted and targeted to individuals in high risk categories.

\section{COMMENTARY}

One third of community dwelling elderly people fall each year, and the incidence increases to one half in those who are institutionalised. About $6 \%$ of falls result in fractures. Frail elderly people, especially those with hip weakness, poor balance, urinary urge incontinence, and polypharmacy, are at high risk. Some falls occur in patients with risk factors that are easily detected, such as visual impairment, poor mobility, disturbed gait, and sedative use. Screening for these factors can lead to early identification of risk and enable successful prevention programmes. However, a recent systematic review identified $>400$ risk factors for falls, which makes screening challenging. ${ }^{1}$

The current guideline by Feder $e t$ al was developed from a systematic review of the literature through $1999 .{ }^{2}$ It delivers evidence based recommendations for healthcare providers caring for elderly people who are living in the community or in residential care. The guideline offers healthcare providers sound advice on preventing falls: we should not advocate exercise alone (with the exception of Thai chi, which improves balance) as a means of preventing falls in unselected elderly people. Rather, we should identify patients at increased risk of falling (and injuries from falls), such as patients who present to the emergency department with a fall and women over 80 years of age who have osteoporosis. These patients should be targeted for multidisciplinary assessment and interventions, including a medical evaluation that encompasses checks for postural hypotension, vision, gait and balance, and medication review, with adjustment or elimination of medications felt to contribute to fall risk. If indicated, I believe that these patients should be referred for occupational and physical therapy and a home safety evaluation. Findings from a recent RCT were not included in the analysis, but they add support to a recommendation advocating hip protectors for nursing home residents and other frail elderly people.

Suzanne D Fields, MD

SUNY at Stony Brook Stomy Brook, New York, USA

1 NHS Centre for Reviews and Dissemination. Preventing falls and subsequent injury in older people. Eff Health Care 1996;2:1-16.

2 Gillespie LD, Gillespie WJ, Cumming R et al. Interventions for preventing falls in the elderly. Cochrane Database Syst Rev 2001;(1):CD000340.

3 Kaannus P, Parkkari J, Niemi S, et al. Prevention of hip fracture in elderly people with use of a hip protector. $N$ Engl $J$ Med 2000;343:1506-13. 